\title{
Learning-by-Catching: Uncertain Invasive-Species Populations and the Value of Information
}

\author{
Sean T. D’Evelyn, Nori Tarui, Kimberly Burnett, James A. Roumasset* \\ Department of Economics, University of Hawaii
}

\begin{abstract}
This paper develops a model of invasive species control when the species' population size is unknown. In the face of an uncertain population size, a resource manager's species-control efforts provide two potential benefits: (1) a direct benefit of possibly reducing the population of invasive species, and (2) an indirect benefit of information acquisition (due to learning about the population size, which reduces uncertainty). We provide a methodology that takes into account both of these benefits, and show how optimal management decisions are altered in the presence of the indirect benefit of learning. We then apply this methodology to the case of controlling the Brown Treesnake (Boiga irregularis) on the island of Saipan. We find that the indirect benefit—-the value of information to reduce uncertainty - is likely to be quite large.
\end{abstract}

Key words: Invasive species, uncertain population, learning, value of information, Brown treesnake 
*Corresponding author. 2424 Maile Way, 542 Saunders Hall, Honolulu, HI 96822.

Email:jimr@hawaii.edu. Phone: 01-808-956-7496, Fax: 01-808-956-4347 


\section{Introduction}

An emerging problem in natural resource policy is how to design efficient strategies for managing invasive species. ${ }^{1}$ Damages from invasive species are ecological as well as economic. These include lost biodiversity and reduced ecosystem services, as well as direct and indirect economic damages such as health damages or lost productivity. Caterpillars from the Asian gypsy moth (Lymantria dispar) cause extensive defoliation, reduced growth and mortality of host trees throughout the northern hemisphere, and hairs on larvae and egg masses lead to allergies in some people. The Nile perch (Lates niloticus) was introduced to Africa's Lake Victoria in 1954 and has since contributed to the extinction of more than 200 endemic fish species through predation and competition for food. Caulerpa taxifolia is a marine alga widely used as a decorative aquarium plant. The alga was accidentally introduced into the Mediterranean Sea in wastewater, where it has now spread over more than 13,000 hectares of seabed. This invader forms dense monocultures that prevent the establishment of native seaweeds and exclude almost all marine life. Tamarisk (Tamarix ramosissima) is a shrubby tree that can be found where its roots reach the water table, such as floodplains, along irrigation ditches and on lake shores. Tamarisk can tolerate a wide range of saline or alkaline soils and is able to dominate floodplain communities in the deserts of the Southwest United States due to its ability to tolerate water stress for extended periods of time. Tamarisk supports few native insects and thus is poor habitat for birds.

\footnotetext{
${ }^{1}$ Invasive species are defined as those plants, animals, and microbes that are nonnative to an area and have caused or have the potential to cause economic and or ecological damage threaten natural resources, biodiversity, and human health worldwide (from President Clinton's Executive Order 13112, signed February 3, 1999).
} 
The well-known invasion of the Brown Treesnake (Boiga irregularis) on the island of Guam poses a real and immediate threat to the state of Hawaii, due to the large and increasing volume of military transport between to the locales, as well as commercial air and sea traffic. The snake has extirpated 11 native bird species on Guam, causes hundreds of hours of power outages a year, and sends a stream of citizens to the hospitals each year to treat venomous snakebites. Eight individual Brown Treesnakes (hereafter, BTS) have been intercepted at the ports in Hawaii, accompanied by hundreds of credible snake sightings resulting in zero captures.

While the economic literature on invasive species has been growing rapidly, most analyses are based on simplifying assumptions that limit their applicability. One aspect of invasive species control that makes practical implementation particularly difficult is that the actual population of the species is almost never known. The only variables that a typical resource manager observes with certainty are the number of the invasive successfully harvested and the effort required to achieve that harvest. Like most renewable resource problems, the literature to date typically assumes a given initial population of the stock of interest. In our paper we develop a model in which the invasive species population is known neither in the initial period nor in subsequent periods. Instead of setting harvest directly, managers set effort levels in each period and then observe the harvest.

In the context of renewable resource use, several studies have analyzed the case where the resource stock is uncertain due to lack of information or measurement error (Clark 
and Kirkwood 1986, Roughgarden and Smith 1996, Sethi et al. 2005). Economic studies of biological invasion have focused on the case of deterministic species population. Some studies analyze special cases where the optimal control under uncertainty is identical to that of the underlying deterministic model (Reed 1979, Knowler 2005). Recent studies analyze uncertain aspects of biological invasion more explicitly. Olson and Roy (2005) examine optimal prevention and control strategies for a randomly introduced biological invasion, assuming that prevention can be effective with certainty and that the population size is observable once invasion occurs. Saphores and Shogren (2005) allow growth to be uncertain, although stock is always accurately observed. We depart from these existing models on invasive species management by assuming uncertain species population size and allow for managers to adjust their subjective probability distributions of population according to catch.

Through species management, the managers obtain new information each period about the probability distribution of the species population size. Borrowing from the literature on renewable resources and learning, we model the connections between observable data (effort and harvest) and the unobservable invasive stock using Bayesian methods. If the effort-harvest function is stochastic but known, the model allows for beliefs about the invasive population to be updated each period and the manager is therefore able to tailor the control strategy appropriately.

Section 2 sets up the baseline case where the population is known with certainty. Section 3 outlines the optimal strategy when the population is uncertain and considers a simplified functional form to characterize the optimal solution. Section 4 illustrates the 
methodology for the case of Brown Treesnake control on the island of Saipan. Section 5 concludes the paper with some suggestions for future research.

\section{Method I: Harvest with observable pest population}

The usual renewable resource problem begins with a resource manager who maximizes the present value of a resource. Optimal management of an invasive species can be approached in a similar fashion. Maximizing the value of invasive species management is the same as minimizing the total present value of the expected costs of removing the species as well as the expected damages caused by the species.

In our model, the invasive species reproduces, causes damages, and is harvested in discrete time periods. We assume that population growth and ecological damages are deterministic, while the manager's harvest of the species is stochastic. The assumption of deterministic growth will help us isolate the information effects we are looking for later in the paper.

Let $X_{t}$ denote the population of the invasive at time $t$. Each period this population causes $d\left(X_{t}\right)$ dollars worth of damage to the local ecosystem. Managers are able to reduce the population through stochastic harvesting. We denote $e_{t}$ as the effort exerted to reduce the population, and $c\left(e_{t}\right)$ the cost the manager bears given $e_{t}$. 
In the case where population is observable each period, the manager is able to set effort decisions based upon the observed harvest. The total present value (or cost, denoted $P V$ ) of the stock of the invasive, $X$, can be thought of as the optimal control costs today, the damages today, and then a discounted sum of the stream of control costs and damages into the future. We denote the discount factor as $\delta$. The harvest in period $\mathrm{t}, h_{t}$, is a function of the stock of the invasive $X_{t}$, the effort level $e_{t}$, and a random variable, $\varepsilon_{t}$. $h_{t}=h\left(X_{t}, e_{t}, \varepsilon_{t}\right)$.

The variable $\varepsilon$ represents the stochastic relationship between harvests, efforts, and population size. Future populations of the invasive will depend on the post harvest population and the species specific growth function, $g$.

$$
X_{t+1}=g\left(X_{t}-h_{t}\right)
$$

The resource manager's objective is to maximize the discounted total expected present value of species management:

$$
E\left\{\sum_{t=0}^{\infty} \delta^{t}\left(-c\left(e_{t}\right)-d\left(X_{t}\right)\right)\right\}
$$

subject to the harvest and species growth constraints (1) and (2) for all $t$ given an initial population size $X_{0}>0$. Given this setup, the following functional equation for stochastic dynamic programming characterizes the optimal solution:

$$
P V\left(X_{t}\right)=\max _{e_{t}}\left\{-c\left(e_{t}\right)-d(X)+\delta E\left[P V\left(X_{t+1}\right)\right]\right\}
$$

subject to (1) and (2) given an initial population size $X_{0}$. Efficiency requires that effort should be chosen so that the marginal cost of the effort is equal to the marginal benefit from reducing the population:

$$
c^{\prime}\left(e_{t}\right)=-E\left[P V^{\prime}\left(X_{t+1} \mid \varepsilon\right) g^{\prime}\left(X_{t}-h_{t}\right) \frac{\partial h\left(X_{t}, e_{t}, \varepsilon\right)}{\partial e_{t}}\right] .
$$


The left-hand side represents the marginal cost of efforts in period $t$. The right-hand side represents the expected marginal benefits of efforts in period $t$. Given $\varepsilon$, an increase in efforts changes harvests, which in turn influences the species growth and the species population next period. The right-hand side captures the resulting change in the expected present value of species control starting next period, where the expectation is taken over $\varepsilon$.

\section{Method II: Harvest under uncertain pest population}

The above problem becomes more interesting and perhaps more realistic when the population of the invasive is not known with certainty. Managers in an uncertain world are no longer able to make policy decisions based on the true population of the invasive, but rather on estimates of those populations. Understandably, as the manager's estimate becomes more accurate, the closer the optimal policy resembles the solution when the population is known.

In the presence of uncertainty, a manager no longer has a simple population of the invasive that they must deal with, but instead is forced to optimize over the belief on uncertain population, i.e., a probability distribution of the possible population size. Every potential population size should be considered when determining the appropriate effort level. 
Let $F$ be the cumulative distribution function that the manager has over the population of the invasive. That is, $F(\mathrm{x})=P(X \leq x)$. Let $f$ be the associated probability density function. The following functional equation for stochastic dynamic programming characterizes the manager's maximization problem:

$$
P V\left(f_{t}\right)=\max _{e_{t}}\left\{-c\left(e_{t}\right)-\int_{0}^{\infty} f_{t}(x) d(x) d x+\delta E\left[P V\left(f_{t+1}\right)\right]\right\} .
$$

In the presence of population uncertainty, the state variable is the probability density function over population size. That is, instead of the actual population, $\mathrm{X}$, the present values are based on the probability distribution of possible populations and expected damages are used in place of actual damages. In what follows we describe how $f_{t+1}$ is determined given $f_{t}$, effort $e_{t}$ and observed harvest $h_{t}$.

\subsection{Bayesian Updating}

Bayesian Updating is a method of determining the distribution derived from previous beliefs and observable data. In this case, the harvest that the managers observe can be used directly in our Bayesian updating framework. First, let us define:

$\tilde{x} \equiv h_{t}+g^{-1}(x)$,

or in other words, $\tilde{x}$ is the population last period that would yield a population of $x$ this period. Now we can write

$$
f_{t+1}\left(x \mid h_{t}, e_{t}, f_{t}(x)\right)=\frac{\pi\left(h_{t} \mid X_{t-1}=\tilde{x}, e_{t}\right) f_{t}(\tilde{x})}{\int_{0}^{\infty} \pi\left(h_{t} \mid X_{t-1}=x^{\prime}, e_{t}\right) f_{t}\left(x^{\prime}\right) d x^{\prime}},
$$


where $\pi$ is the probability of observing a harvest of exactly $h_{t}$ given population size $\tilde{x}$ and an effort level $\mathrm{e}_{\mathrm{t}}$.

Equations (6) and (8) together yield the necessary equations for maximizing the present value of the invasive under uncertainty. Since effort appears in the right hand side of equation (8), effort will not only affect the expected mean of the population estimate next period, but may also affect the spread. When the posterior distribution given a harvest level is second-order stochastically dominated by the posterior given a lower harvest level, effort becomes more valuable than in the perfect certainty case. This extra value comes from the value of information.

\subsection{A Specific Functional Form}

The equations presented in the last section describe how a resource manager would optimally determine the level of effort in each period. In order to provide a more precise understanding of how harvest and information updating work in this framework, we will add three simplifying assumptions to our model. First, we assume damages are linear in population. With this assumption we can set $d\left(X_{t}\right)=d X_{t}$ for all $X_{t}$. Secondly, we assume an exponential growth rate of the invasive, allowing us to write $g\left(X_{t}-h_{t}\right)$ as $k X_{t}-k h_{t}$.

Finally, we assume that control efforts are a binomial process, where each member of the population has an equal likelihood of being caught on a given round of treatment.

While these assumptions are not equally applicable to all invasive species, they can prove useful in a variety of settings. For example, while most managers agree that invasive 
species can cause massive economic damages, determining how much damage each individual member of the species causes can be a great challenge. For this reason, approximating damages as a linear function of the invasive population may be a reasonable estimate.

Not all species reproduce at an exponential rate. Resource economics typically assumes a logistic growth function, where the rate of growth slows as the population increases, to the point where annual growth rate declines past the point of maximum sustainable yield. However, invasive species introduced to a new ecosystem usually have ample supply of prey-base and room to expand. The ability to rapidly multiply in number is one factor that makes invasive species such a problem in many cases, and makes an exponential growth function more reasonable.

Our most restrictive assumption is the availability of a binomial control strategy. In order for a control process to qualify it must have a repeatable methodology, with a likelihood of successful harvest of a given member in any iteration that is independent both of the harvest of other members of the population as well as independent of past control activities. This would exclude species where a large percentage of the population could be captured all at once (such as pack animals) or any control strategy that systematically cleared an area of the pest.

However, this approach is defendable for a wide variety of species, even ones that may not seem applicable. One example is that of sessile species such as the noxious weeds 
that threaten agriculture. If each sweep of an area is considered one round of the binomial event, so long as there is an imperfect chance of finding a given weed on a particular round, then frequency of the sweeps may depend on expected size of the population. For example, suppose a manager attempts to rid the managed area of spotted knapweed. The manager knows that it will be difficult to get $100 \%$ of the knapweed on the first sweep of the area. The assumption is that managers can capture a higher percentage of the knapweed with either repeated sweeps or more intense sweeps at the same effectiveness per person-hour.

As for animals that could potentially be caught at one time, thus violating the independence assumption, the problem can be alleviated by changing the unit of analysis. For example, red imported fire ants are likely to live together in anthills, and thus if one fire ant is discovered, it will be likely that a whole colony will be controlled. However, if the unit of analysis is moved to controlling anthills (or queen ants) then the harvest of each of those units are more likely to be independent of each other.

Effort in this context is a function of control techniques such as the number of snake traps set, concentration of toxicants, or simply person-hours spent hunting. These techniques are able to capture, trap, or otherwise kill a percentage of the invasive population in one period. Furthermore, varying either the frequency or the intensity of these techniques allows the manager to set different percent capture rates. It is these expected percent capture rates that we will call $e$ in this context. ${ }^{2}$ For example, an $e=.95$ would mean that

\footnotetext{
${ }^{2}$ We use expected capture rate as our measure of effort rather than the control technique itself, as is commonly done in resource economics (e.g., measuring effort as number of boats or person-hours). This
} 
the manager expected to capture $95 \%$ of the invasives whatever the optimal combination of control techniques necessary. The actual percent of the population captured varies around $e_{t}$, but harvesting can never add to the population nor can it remove more of a species than are actually present.

In addition to the plausibility and the analytical convenience explained above, this framework might be consistent with the decision making by the real world managers, who generally set effort levels for a given period and then simply harvest whatever that effort level yields. This setting is in contrast to many previous resource models where, even in the face of uncertainty, managers set a planned harvest level (or sometimes escapement level) and then merely observe whether or not that harvest depletes the population.

Given effort level $e$ and population $X$, the probability that exactly $h$ individuals are caught is given by:

$$
\frac{X !}{h !(X-h) !} e^{h}(1-e)^{X-h}
$$

However, this equation only works for discrete values of $h$ and $X$. Where convenient we will use the continuous approximation of the binomial distribution. As $X$ increases in size, and therefore as it approaches a continuous variable, the distribution of $h$ becomes:

allows the transition of population from one period to the next to simply be the previous population minus the proportion of snakes removed. Otherwise, the previous population would have to be reduced by a more complicated function of the control technique. For example, we would need to specify the precise relationship between the control technique and the number of individuals removed. In the case of snakes, for example, this would require specification of how many snakes would be removed through traps or search hours. Though this relationship still needs to be specified in our case, converting to capture rate simplifies the equation of motion. This substitution greatly facilitates computation in the case of harvest under uncertainty. 
$h \sim N\left(e_{t} X_{t}, X_{t} e_{t}\left(1-e_{t}\right)\right)$.

Separating harvest into its deterministic and stochastic components, we have:

$h=e_{t} X_{t}+\varepsilon_{t}$, where $\varepsilon_{t} \sim N\left(0, X_{t} e_{t}\left(1-e_{t}\right)\right)$.

Let the expected proportion of the population controlled by a single unit of control effort be denoted $\alpha$. If the effectiveness of each unit is independent, the expected proportion captured after $n$ units are used is denoted:

$e=\left(1-(1-\alpha)^{n}\right)$

When characterizing the cost function, we assume that costs are linear in the control activities that determine $e$. If the cost per unit of the removal technique is $\mathrm{c}$, then the cost of effort is:

$c\left(e_{t}\right)=\frac{c}{\log (1-\alpha)} \log \left(1-e_{t}\right) \equiv \theta \log \left(1-e_{t}\right)$.

Our problem can now be characterized by the following stochastic dynamic programming equation:

$$
\begin{aligned}
& P V\left(f_{t}\right)=\max _{e_{t}}\left\{-c\left(e_{t}\right)-\int_{0}^{\infty} f_{t}(x) d(x) d x+\delta E\left[P V\left(f_{t+1}\right)\right]\right\}, \\
& \hat{h}_{t}=X_{t} e_{t}\left(X_{t}\right)+\varepsilon_{t} \\
& h_{t}=\max \left(0, \min \left(\hat{h}_{t}, X_{t}\right)\right) \\
& X_{t+1}=k X_{t}-k h_{t} \\
& \varepsilon \sim N\left(0, X_{t} e_{t}\left(1-e_{t}\right)\right)
\end{aligned}
$$


where $\hat{h}_{t}$ is potential harvest and the probability density function $f_{t}$ is updated according to equation (8). Noting that $\hat{h}_{t}$ cannot exceed current population nor can it be negative), $h_{t}$ is thus realized harvest.

A first order necessary condition for efficient management in this problem is:

$$
\frac{\theta}{1-e_{t}}+\delta \frac{\partial E\left[P V\left(f_{t+1}\right)\right]}{\partial e_{t}}=0
$$

where the marginal cost of removal today must be equal to the present value of the change in damages and effort in later periods. This condition describes optimal effort, and thus control of an invasive species when harvesting under uncertainty.

\subsection{Characterizing the optimal solution}

If the harvest technique is a binomial process and the expected percent capture rates that efforts engenders are known, then the population can be estimated through the observed harvest and effort rates. We use the normal approximation for the binomial as given above wherever convenient.

If the manager has no prior knowledge about the stock of the invasive population at time $t=0$, after observing effort they can calculate:

$E\left[h_{0}\right]=E\left[e_{0} X_{0}+\varepsilon_{0}\right]=e_{0} E\left[X_{0}\right]$.

Thus, after observing $h$ and $e$, an unbiased estimate of $X_{t}$ is given by:

$E\left[X_{0}\right]=\frac{h_{0}}{e_{0}}$ 
Note that equation (21) makes intuitive sense. If the manager expected to capture $50 \%$ of the population and then 100 species are successfully caught, a reasonable estimate for the total population would be 200 . This estimate is approximately normally distributed with variance:

$V\left[E\left[X_{0}\right]\right]=V\left[\frac{h_{0}}{e_{0}}\right]=\frac{1}{e_{0}^{2}} V\left[h_{0}\right]=\frac{X_{0}\left(1-e_{0}\right)}{e_{0}}$.

This information is only somewhat useful in its current state, as population estimates at time $t$ can only be obtained after the harvest for that time period takes place. However, since our growth function is known, we can use data gathered in the previous period to estimate the next period's population before any harvest decision is made. We define $M_{t}$ as the pre-harvest estimate of $X_{t}$ such that:

$M_{1}=E\left[X_{1}\right]=E\left[k X_{0}-k h_{0}\right]=k \frac{h_{0}}{e_{0}}-k h_{0}=k h_{0} \frac{1-e_{0}}{e_{0}}$.

The variance of this estimate is:

$V\left[M_{1}\right]=V\left[E\left[k X_{0}-k h_{0}\right]\right]=k^{2} V\left[E\left[X_{0}\right]=\frac{k^{2} X_{0}\left(1-e_{0}\right)}{e_{0}}\right.$.

With this framework, we now have a means of deriving current population estimates from easily observable data. Unfortunately, the variance of these estimates does depend on the true stock size, an unknown parameter for the managers. In order to maintain normality and simplify our results, in our analysis we make the assumption that the managers know the variances of their estimates with certainty. Realistically, these variances would have to be estimated imprecisely, but would likely yield similar results. 
Continuing with our example, we now have a pre-harvest expected population for period one. With this information, the manager is able to make a more informed decision for second period effort levels. However, after harvesting a second time, the manager could now form a second estimate for the population at time one from this new information. This new estimate can be written as:

$$
x_{1}=E\left[X_{1}\right]=\frac{h_{1}}{e_{1}},
$$

with variance $V\left[x_{1}\right]=E\left[X_{1}\right]=\frac{X_{1}\left(1-e_{1}\right)}{e_{1}}$.

Except in very rare cases, this new estimate, $\mathrm{x}$, will not equal the previous estimate of $X_{1}$. Because both of these estimates are normally distributed, obtaining the distribution for the most likely estimate for $X_{1}$ is straightforward. We first define $\rho_{t}$ to be the precision of the estimate $\mathrm{M}$, equal to $1 / \mathrm{V}\left[M_{t}\right]$ and $\mathrm{p}$ to be the precision of our single period estimate $\mathrm{x}$, equal to $1 / \mathrm{V}[\mathrm{x}]$. Using these parameters, we can create a new estimate for $X_{1}$ that uses the available information to its fullest. As can be found in any elementary statistics book, the new estimate using both sets of information becomes: $\frac{M_{1} \rho_{1}+x_{1} p_{1}}{\rho_{1}+p_{1}}$

where the variance is:

$$
\frac{1}{\rho_{1}+p_{1}}
$$

Generalizing this, and applying it to the problem of interest, the manager's problem becomes:

$$
P V\left(M_{t}, \rho_{t}\right)=\max _{e_{t}}\left\{-\theta \log \left(1-e_{t}\right)-d M_{t}+\delta E\left[P V\left(M_{t+1}, \rho_{t+1}\right)\right]\right\},
$$




$$
\begin{aligned}
& \hat{h}_{t}=X_{t} e\left(M_{t}, \rho_{t}\right)+\varepsilon_{t}, \\
& h_{t}=\max \left(0, \min \left(\hat{h}_{t}, X_{t}\right)\right), \\
& X_{t+1}=k X_{t}-k h_{t}, \\
& \varepsilon \sim N\left(0, X_{t} e_{t}\left(1-e_{t}\right)\right), \\
& M_{t+1}=k \frac{\left(M_{t}-h_{t}\right) \rho_{t}+\left(x_{t}-h_{t}\right) p_{t}}{\rho_{t}+p_{t}}, \\
& \rho_{t+1}=\frac{\rho_{t}+p_{t}}{k^{2}}, \\
& x_{t}=\frac{h_{t}}{e_{t}}, \\
& p_{t}=\frac{e_{t}}{X_{t}\left(1-e_{t}\right)} .
\end{aligned}
$$

where $\hat{h}_{t}$ and $h_{t}$ are again potential and realized harvest as described in the previous section, $X_{t+1}$ the new (uncertain) population, $M_{t+1}$ the pre-harvest estimate of $X_{t+1}, \rho_{t+1}$ the precision of the new pre-harvest estimate, $x_{t}$ the estimate of the population using only harvest and effort in time $t$, and $p_{t}$ the precision of this estimate.

In this uncertain framework, effort decisions can only be made based on the expected population $M$ and the precision with which we can make that estimate, $\rho$. The present value is thus not only a function of the stock of the invasive, but also of the information held at time $t$. Our first order equation now becomes:

$$
\frac{\theta}{1-e_{t}}+\delta \frac{d E\left[P V\left(M_{t+1}, \rho_{t+1}\right)\right]}{d e_{t}}=0
$$


or

$\frac{-\theta}{1-e_{t}} \approx-\delta k E\left[X_{t} P V_{1}\left(M_{t+1}, \rho_{t+1}\right)\right]+\frac{\delta}{k^{2}\left(1-e_{t}\right)^{2}} E\left[\frac{P V_{2}\left(M_{t+1}, \rho_{t+1}\right)}{X_{t}}\right]$

The term $P V_{1}\left(M_{t+1}, \rho_{t+1}\right)$ refers to the partial derivative of the present value with respect to the first argument, $\mathrm{M}_{\mathrm{t}+1}$, and $P V_{2}\left(M_{t+1}, \rho_{t+1}\right)$ the partial derivative with respect to the second term, $\rho_{t+1}$. Expected values are taken both over all possible stocks of the invasive, $X$, and over all possible harvest levels, $h$, given $X$. The first term on the right hand side of the equation is equivalent to the right hand side of equation (19) and represents the marginal benefit from reducing the population in the next period.

Effort in the context of uncertainty now gains a secondary benefit. Not only does it reduce the expected population next period, but it also increases the precision with which the manager knows the population. The second term on the right hand side of equation (39) represents the additional value gained from increasing the precision of the population estimate in the next period. The value of this term is the additional value above and beyond the damages avoided from removing the invasive species from the existing population, one almost always ignored in the literature to date.

\section{Case Study: Brown Treesnake on the Island of Saipan}

In order to illustrate the applicability of a population updating approach, we now turn to the case of the BTS on Saipan, located north of Guam in the Commonwealth of the 
Northern Mariana Islands. BTS has already been noted to cause extensive economic damage on Guam in the form of biodiversity losses, massive power outages, and health costs (Savidge 1987, Fritts et al. 1987, 1990, 1994). Because of the heavy military presence on Guam and its accompanying movement of people and cargo off island, BTS threatens surrounding islands in the Pacific. A recent estimate of an optimally managed BTS population in Hawaii, for example, approaches \$18 million dollars (Burnett 2007). Although Guam authorities have tried vigilantly to minimize the chance of BTS escaping to other islands, Saipan has had several sightings of BTS, and program scientists and managers believe there is an incipient population (Nathanial Hawley, personal communication).

Based on data gathered on Guam, the cost to sweep the island looking for snakes enough times to obtain an expected percent capture rate of e is: $-\$ 2,023,000 * \log (1-e)$. For a more detailed look at the derivation of this formula, see the appendix. Assuming that BTS will cause similar damages to Saipan as it might in Hawaii, we estimate $d$ to be 121.79 per year or approximately $\$ 10.15$ per month ${ }^{3}$. Since prey base is likely to also be similar to that of Guam, we estimate a per month growth rate of 1.0399, and we set a monthly discount rate of 1.00165 , which is equivalent to an annual discount rate of $2 \%$.

Conversations with invasive species managers suggest that there is a small incipient population of BTS on Saipan. There have been 75 credible sightings of BTS on island, leading to zero captures. While it is possible that all 75 sightings could be due to a single snake, this is highly unlikely. For the purposes of this example, we suppose that the

\footnotetext{
${ }^{3}$ See Burnett (2007) for a detailed description of damage estimations.
} 
manager estimates that there are about 200 snakes, with a standard deviation of 100 (and therefore a precision of .0001). Even if the true population is drastically different from 200 , the updating process will quickly yield a better estimate.

In order to illustrate the effects of learning on the optimal species management, we conducted a Monte Carlo simulation and compared two strategies —one with learning $\left(S^{L}\right)$ and the other without learning $\left(S^{N}\right)$. With strategy $S^{L}$, the manager updates its belief on the probability distribution of the population size based on efforts and observed harvests. Strategy $S^{N}$ assumes that the manager chooses efforts in each period based on the initial belief about the species distribution without updating the belief over time.

We assume the same initial belief about the distribution of population size: $X_{0} \sim N\left(M_{0}, 1 / \rho_{0}\right)$, where $\left(M_{0}, \rho_{0}\right)$ is specified as in the above section. Under strategy $S^{N}$, the manager chooses

$$
e_{t}^{N}=\max \left\{0,1-\frac{X^{*}}{k M_{t}}\right\}, t=0,1,2, \ldots, T,
$$

where $X^{*}$ represents the steady-state species population size in the deterministic version of the model (where the initial population is given by $M_{0}$ ) and where

$$
M_{t+1}=k M_{t}\left(1-e_{t}^{N}\right), t=0,1,2, \ldots, T,
$$


given $M_{0}{ }^{4}$ Strategy $S^{N}$ is the optimal solution for the deterministic case

$\left(M_{t} \equiv X_{t}, \rho=+\infty, \varepsilon_{t} \equiv 0\right)$. The true population dynamics follows equations (30)-(33)

where $e_{t}$ is replaced by $e_{t}^{N}$.

Under strategy $S^{L}$, the manager chooses

$e_{t}^{L}=\max \left\{0,1-\frac{X^{*}}{k M_{t}}\right\}, t=0,1,2, \ldots, T$,

where the mean and the precision $\left(M_{t}, \rho_{t}\right)$ are updated according to the Bayes rule described in equations (34), (35). Though this strategy with learning is not necessarily the optimal solution to the species management problem (29)-(37), it incorporates learning in a simple and practically relevant manner. ${ }^{5}$ Given the resource managers' information and costs constraints, a relatively simple strategy such as $S^{L}$ might be implemented more easily than an optimal strategy.

We set the time horizon $T$ to be 240 (i.e., 20 years), and computed the present values of species control under the two strategies by running 1,000 replications. We evaluate the outcome of each trial using the social costs of invasive species management, i.e. the present value of the sum of control costs and damages due to invasion given the realization of initial population size, with and without learning. Let $S C_{n}^{L}$ and $S C_{n}^{N}$ be the realized social costs with and without learning under the $n$th replication (trial):

\footnotetext{
${ }^{4}$ Given the parameter values specified in the previous section, $X^{*}$ equals 329 .

${ }^{5}$ The effort decision in equation (43) is an example of "constant escapement policy." When a constant escapement policy is optimal in a deterministic resource-use model, it is not necessarily optimal in the same model with uncertainty (Clark and Kirkwood 1986, Sethi et al. 2005). For conditions under which equivalence obtains, see Reed (1979).
} 
$S C_{n}^{J}=\sum_{t=0}^{T} \delta^{t}\left\{-c\left(e_{t}^{J}(n)\right)-d\left(X_{t}^{J}(n)\right)\right\}, \quad J=L, N$

Let $\overline{S C}^{L}$ and $\overline{S C}^{N}$ be the average of social costs over all trials $n=1, \ldots, T$. The difference between $\overline{S C}^{L}$ and $\overline{S C}^{N}$ indicates the expected value of information.

Out of 1,000 trials, $S C^{L}$ exceeded $S C^{N}$ only 41 times. The 1,000 trials yielded the averages $\overline{S C}^{L}=\$ 13.6$ million with learning and $\overline{S C}^{N}=\$ 78.9$ million without learningor about 6 times as large as $\overline{S C}^{L}$. This experiment implies that the value of information through species control can be substantial. Under learning, updated information allows the managers to change the flexibly of control efforts. Such flexible updating allows the manager to achieve a smaller expected social cost.

Figures 1 and 2 describe the population dynamics and the effort profiles for two illustrative trials. In each figure, the top panel describes the true species population dynamics while the bottom panel shows the time profile of effort. In both cases, the effort profile with learning fluctuates constantly while the effort profile without learning is monotonic over time. This is because, under learning, the probability distribution of population is constantly updated. During periods of high and low catch, the estimated population adjusts up and down accordingly, triggering a higher (lower) effort level in the next period. In contrast, the effort profile without learning is monotonic because the estimated population remains unchanged. Figure 1 plots a scenario where strategy $S^{N}$ resulted in extinction while strategy $S^{L}$ caused the population size to fluctuate around $X^{*}$ 
$=329$, the optimal steady state for the deterministic version of the model. Despite species extinction, $S^{N}$ resulted in a higher social cost $\left(8 \%\right.$ larger than $\left.S C^{L}\right)$ due to an excess of control costs resulting from the manager failing to learn about the diminished population. Figure 2 demonstrates a contrasting case wherein control without learning results in insufficient effort and an explosion of the pest population. In this trial, $S C^{N}$ was about $540 \%$ larger than $S C^{L}{ }^{6}$ Together, the two figures demonstrate that failure to learn may result in control efforts that are too high or too low. These two trials imply that, given exponential growth of an unchecked pest population, the cost of applying insufficient control efforts is much larger in magnitude than the cost of applying excessive control efforts relative to what Bayesian learning indicates.

[Figure 1 here]

[Figure 2 here]

\footnotetext{
${ }^{6}$ This large number is due to the assumption that, without learning, the manager does not update her belief about the population, even if the population explodes. This assumption is standard in the value-ofinformation literature, and may be a reasonable baseline case for valuing information in other contexts such as weather forecasting (e.g., Costello et al. 1998). In the context of invasive species management with exponential population growth, this assumption of a naïve resource manager might not be realistic. At some point, the manager is likely to learn that her population beliefs are wrong, but when? Several scenarios can be considered, but see Burnett et al.'s (2007) demonstration in another context that the losses from delayed correction of status quo policies (as opposed to no correction) are still large. Note also that in the 568 in which social costs of failing to learn are modest (because the population is actually declining) the value of learning is still $17 \%$.
} 


\section{Discussion and Conclusion}

Optimal management of an invasive species is challenging when the actual population being controlled is uncertain. How should resource managers take account of existing information about population size and invest in learning about that size? In this paper, we develop a framework to solve for optimal invasive species management where the resource manager can update their subjective belief about species population using Bayesian methods. When effort spent on controlling the population not only lowers future expected population but also provides the manager with more precise estimates for the future, early control efforts become even more important than in models where invasive populations are known. This result is derived analytically and illustrated numerically.

By comparing the average present values of invasive species management with and without learning, we can compute the value of additional information due to learning. We parameterize our model to analyze BTS control in Saipan and estimate the value of information. A Monte Carlo simulation indicates that learning reduces the cost of species control and damages in almost all cases. In section 4 we showed the result of two trials where, in the absence of learning, one resulted in excessive control efforts and costly species eradication while the other resulted in insufficient control efforts and species population explosion. On average, the social cost with learning was only $1 / 6$ of the cost without learning. Costs are especially high in the case where the population has been 
underestimated and where the population grows rapidly in the face of under-control. The value of learning will be lower if the planner merely delays adjusting the control efforts upwards but substantial nonetheless. These results suggest that a traditional framework to analyze biological invasion given deterministic species population will not provide efficient management strategies, and that the value of information due to learning can be large.

The model developed in this paper can be extended in a number of directions. Population estimation through stochastic harvesting processes is useful in any setting, but it is even more useful when either growth is uncertain or when the populations are subject to random shocks (e.g. due to new invasions, a new food source, disease, or a new predator). While estimation techniques doubtlessly become less precise in such cases, continual updating of the population is even more important. Another extension could allow for heterogeneity in catchability, such that the more easily caught tend to be caught earlier. This would imply a role for learning about the efficacy of control techniques as well as learning about the size of the population. Analysis of these issues are left for future research.

\section{Acknowledgements}

We thank the editor Ram Ranjan and two anonymous referees for their helpful comments. 


\section{References}

K. Burnett, Optimal Prevention and Control of Invasive Species: The Case of The Brown Treesnake, Ph.D. Dissertation, University of Hawaii at Manoa (2007). http://www2.hawaii.edu/\%7Ekburnett/Diss.pdf

K. Burnett, B. Kaiser, and J. Roumasset, Economic Lessons from Control Efforts for an Invasive Species: Miconia calvescens in Hawaii (forthcoming, Journal of Forest Economics) (2007).

K. Burnett, B. Kaiser, B. Pitafi and J. Roumasset, Prevention, Eradication, and Containment of Invasive Species: Illustrations from Hawaii, Agricultural and Resource Economics Review 35 (2006)(1), pp. 63-77.

C. W. Clark and G. P. Kirkwood, On uncertain renewable resource stocks: optimal harvest policies and the value of stock surveys. Journal of Environmental Economics and Management 13 (1986), pp. 235-244.

C. J. Costello, R. M. Adams and S. Polasky, The Value of El Nino Forecasts in the Management of Salmon: A Stochastic Dynamic Assessment. American Journal of Agricultural Economics 80 (1998), pp. 765-777.

T.H. Fritts, N.J. Scott and J.A. Savidge, Activity of the arboreal browntree snake (Boiga irregularis) on Guam as determined by electrical outages, The Snake 19 (1987), pp. 51-8.

T.H. Fritts, M.J. McCoid and R.L. Haddock, Risks to infants on Guam from bites of the brown tree snake (Boiga irregularis), American Journal Tropical Medicine and Hygiene 42 (1990), pp. 607-11.

T.H. Fritts, M.J. McCoid and R.L. Haddock, Symptoms and circumstances associated with bites by the browntree snake (Colubridae: Boiga irregularis) on Guam, Journal of Herpetology 28 (1994), pp. 27-33.

D. Knowler, Reassessing the Costs of Biological Invasion: Mnemiopsis leidyi in the Black Sea, Ecological Economics 52 (2005) (2), pp. 187-199.

L.J. Olson and S. Roy, On Prevention and Control of an Uncertain Biological Invasion, Review of Agricultural Economics 27 (2005) (3), pp. 491-497.

W. Reed, Optimal Escapement Levels in Stochastic and Deterministic Harvesting Models, Journal of Environmental Economics and Management 6 (1979), pp. 350-363.

J. Roughgarden and F. Smith, Why Fisheries collapse and what to do about it, Proceedings of the National Academy of Sciences 93 (1996), pp. 5078-5083. 
J. D. Saphores and J. F. Shogren, Managing Exotic Plants Under Uncertainty: Optimal Control Actions and Bioeconomic Investigations, Ecological Economics 52 (2005) (3), pp. 327-340.

J. A. Savidge, Extinction of an island forest avifauna by an introduced snake, Ecology 68 (1987), pp. 660-668.

G. Sethi, C. Costello, A. Fisher, M. Hanemann and L. Karp, Fishery management under multiple uncertainty, Journal of Environmental Economics and Management 50 (2005) (2) 300-318. 


\section{Appendix: Cost of removal}

In a recent study, Gordon Rodda of U.S. Geological Survey examined the catchability of snakes on a 5 hectare enclosed plot in Guam. Although a large degree of individual heterogeneity was found, BTS in the experiment had an average of a $16.36 \%$ chance of being caught on a given night of trapping. Saipan is approximately 12,043 hectares in area, all of which is potential snake habitat. With the aid of Gordon Rodda, we estimate the cost per night of trapping per 5 hectare plot to be around $\$ 150$. Thus, using the definition of $\theta$, above, our costs of effort on Saipan will be:

$\frac{12,043 \text { ha. }}{5 \text { ha./plot }} * \$ 150 /$ plot $* \frac{1}{\log (.163564)} * \log (1-e) \approx-\$ 2,023,177 \log (1-e)$. 



$\mathrm{X}^{*}$ is the optimal steady state for the deterministic version of the model where initial population equals the mean of the initial belief.

Figure 1. Species population dynamics and effort: case I 



$\mathrm{X}^{*}$ is the optimal steady state for the deterministic version of the model where initial population equals the mean of the initial belief.

Figure 2. Species population dynamics and effort: case II 